\title{
Escherichia coli acid resistance: CAMP receptor protein and a 20 bp cis-acting sequence control pH and stationary phase expression of the gad $A$ and gadBC glutamate decarboxylase genes
}

\author{
Marie-Pierre Castanie-Cornet $†$ and John W. Foster
}

Author for correspondence: John W. Foster. Tel: +1 3344606323. Fax: +1 3344607931.
e-mail: fosterj@sungcg.usouthal.edu

Department of

Microbiology and

Immunology, University of

South Alabama College of

Medicine, Mobile,

AL 36688, USA
Acid resistance is an important feature of both pathogenic and non-pathogenic Escherichia coli. It enables survival in the acidic regions of mammalian gastrointestinal tracts and is largely responsible for the small number of bacteria required for infection/colonization. Three systems of acid resistance have been identified, the most efficient of which requires glutamic acid during pH 2 acid challenge. Three proteins associated with glutamate-dependent acid resistance have been identified. They are glutamate decarboxylase (encompassing two isozymes encoded by gadA and gadB) and a putative glutamate: $\gamma$-amino butyric acid antiporter (encoded by gadC). The results confirm that the GadA and GadB proteins increase in response to stationary phase and low environmental $\mathrm{pH}$. The levels of these proteins correspond to concomitant changes in gadA and gadBC mRNA levels. Fusions between lacZ and the gadA and gadBC operons indicate that this control occurs at the transcriptional level. Western blot, Northern blot and fusion analyses reveal that regulation of these genes is complex. Expression in rich media is restricted to stationary phase. However, in minimal media, acid pH alone can trigger induction in exponential or stationary phase cells. Despite this differential control, there is only one transcriptional start site for each gene. Expression in rich media is largely dependent on the alternate sigma factor $\sigma^{5}$ and is repressed by the CAMP receptor protein (CRP). In contrast, $\sigma^{\text {s }}$ has only a minor role in gad transcription in cells grown in minimal media. Deletions of the regulatory region upstream of gadA provided evidence that a $20 \mathrm{bp}$ conserved region located $50 \mathrm{bp}$ from the transcriptional start of both operons is required for expression.

Keywords: acid resistance, glutamate decarboxylase, $\mathrm{pH}$ control, Escherichia coli, rpoS

\section{INTRODUCTION}

Escherichia coli, in its natural environment, endures many different stress conditions including frequent periods of acid stress. Even though the organism prefers a neutral $\mathrm{pH}$ for growth, E. coli can tolerate extremely

† Present address: Laboratoire d'Ingéniérie et Dynamique des Systèmes Membranaires, Centre National de la Recherche Scientifique, 31 chemin Joseph Aiguier, 13402 Marseille cedex 20, France.

Abbreviation: CRP, CAMP receptor protein. acidic conditions ( $\mathrm{pH} 2-3$ ) for several hours (Gorden \& Small, 1993; Waterman \& Small, 1996). This is particularly important for the pathogenic E. coli that generally must survive the strong acid $\mathrm{pH}$ of the stomach to cause disease. We have previously shown that three distinct acid-resistance systems are involved in protecting cells from acidic shock (Castanie-Cornet et al., 1999; Lin et al., 1995, 1996). These systems are active only in the stationary phase of growth. Acid-resistance system 1 relies directly on $\sigma^{\mathrm{S}}$, the stationary-phase sigma factor (Lange \& Hengge-Aronis, 1991; Loewen \& 
Table 1. Bacterial strains used in this study

\begin{tabular}{|c|c|c|}
\hline Strain & Genotype & Source or reference \\
\hline EK227 & E. coli K-12 wild-type & A. C. Matin* \\
\hline EK298 & $\begin{array}{l}\text { (TE2680) } \mathrm{F}^{-} \lambda^{-} \text {IN }(r r n D-r r n E) \Delta(l a c) X 74 \text { rpsL } \\
\text { galK2 recD1903:: Tn10dTc trpDC::putPA1303 }\end{array}$ & T. Elliott $†$ \\
\hline EK344 & GE1050 $\Delta c r p:: C m$ & G. Weinstock $\ddagger$ \\
\hline EF362 & K-12 rpos : : Tn10 & Castanie-Cornet et al. (1999) \\
\hline EF614 & $\begin{array}{l}\text { EK298 trpDC::putPA1303-Km-gadB }{ }^{-203}: \text { : lacZ } \\
\text { (o)(-203 to }+788 \text { fusion })\end{array}$ & $\mathrm{EK} 298 \times \mathrm{pCR} 384$ \\
\hline EF615 & $\begin{array}{l}\text { EK298 trpDC::putPA1303-Km-gad } A^{-165}: \text { : lacZ } \\
\text { (o) }(-165 \text { to }+788 \text { fusion })\end{array}$ & EK298 $\times$ pCR385 \\
\hline EF640 & Tet $^{\mathrm{s}}$ derivative of EF615 & \\
\hline EF647 & $\begin{array}{l}\text { EK298 trpDC::putPA1303-Km-gadA }{ }^{-165}:: \text { lacZ } \\
\text { (o) rpoS::Tn10 }\end{array}$ & $\mathrm{EF} 640 \times \mathrm{EF} 362$ \\
\hline EF660 & Tet $^{\mathrm{s}}$ derivative of EF647 & \\
\hline EF663 & $\begin{array}{l}\text { EK298 trpDC::putPA1303-Km-gadA } A^{-51}:: \text { lac } \\
\text { (o) }(-51 \text { to }+788 \text { fusion })\end{array}$ & EK298 $\times$ pCF402 \\
\hline EF666 & $\begin{array}{l}\text { EK298 trpDC::putPA1303-Km-gad } A^{-86}: \text { :lacZ } \\
\text { (o) }(-86 \text { to }+788 \text { fusion })\end{array}$ & $\mathrm{EK} 298 \times$ pCF403 \\
\hline EF676 & $\begin{array}{l}\text { EK298 trpDC::putPA1303-Km-gadA }{ }^{-165}:: l a c Z \\
\text { crp:: Cm }\end{array}$ & $\mathrm{EF} 615 \times \mathrm{EK} 344$ \\
\hline EF677 & $\begin{array}{l}\text { EK298 trpDC::putPA1303-Km-gadA }{ }^{-165}:: \text { lacZ } \\
\text { rpoS::Tn10 crp:: Cm }\end{array}$ & EF647 × EK344 \\
\hline
\end{tabular}

* Stanford University, CA, USA.

† West Virginia University, Morgantown, WV, USA.

‡ University of Texas Medical School, Houston, TX, USA.

Hengge-Aronis, 1994), and will protect cells at $\mathrm{pH} 2.5$ in minimal media. The two other systems involve specific amino-acid decarboxylases and rely on exogenously supplied glutamate (acid-resistance system 2) or arginine (acid-resistance system 3) for protection during acidic shock in minimal media. These systems are thought to provide acid resistance by consuming intracellular protons via the amino acid decarboxylation reaction and also by gradual alkalinization of the medium.

Two operons, gadA and gadBC, have been identified as participating in the glutamate-dependent acid-resistance system 2 (Castanie-Cornet et al., 1999; Hersh et al., 1996). The gadA and gadB genes encode highly homologous glutamate decarboxylase isoforms (Smith et al., 1992), whereas gadC encodes a membrane-associated glutamate $\gamma \gamma$-amino butyric acid (GABA) antiporter that exchanges exogenous glutamate for intracellular GABA. Glutamate decarboxylase production has been shown to increase in response to acid, osmotic and stationary phase signals (Castanie-Cornet et al., 1999; De Biase et al., 1999). De Biase et al. (1999) have shown that the histone-like protein $\mathrm{HN}-\mathrm{S}$ acts as a negative regulator of gad expression and reported that both operons are only induced in stationary phase, with enhanced expression occurring at acid $\mathrm{pH}$ (De Biase et al., 1999). Expression was reported to be totally dependent on $\sigma^{\mathrm{S}}$ and was not observed in exponential phase cells. However, glutamate-dependent acid resistance, which requires $\mathrm{Gad}$, does not depend on $\sigma^{\mathrm{S}}$ (Castanie-Cornet et al.,
1999; Lin et al., 1995). Western blot analyses conducted in our laboratory have revealed that acid induces GadA and GadB production in exponential phase cells grown in minimal media even in rpoS mutants devoid of $\sigma^{\mathrm{s}}$ (Castanie-Cornet et al., 1999). A reasonable hypothesis that would account for these apparently conflicting results is that multiple regulatory factors influence $\mathrm{gad}$ expression and that growth in complex or minimal glucose media determines which regulator dominates. We now provide evidence that gad transcription is controlled by several additional factors and that these factors differ depending upon the growth phase and environment. The factors involved include $\sigma^{\mathrm{S}}$, the cAMP receptor protein (CRP), $\sigma^{70}$ and a $20 \mathrm{bp}$ conserved region of the gad operon.

\section{METHODS}

Bacterial strains and culture media. The bacterial strains used in this study are listed in Table 1 . E. coli was grown at $37^{\circ} \mathrm{C}$ in either minimal $\mathrm{E}$ medium (Vogel \& Bonner, 1956), E medium containing $0.4 \%$ glucose $(E G)$ or in complex medium Luria-Bertani broth (LB) buffered with either $100 \mathrm{mM}$ MES (pH 5.5) or MOPS (pH 8). Antibiotics were used at the following concentrations : ampicillin, $50 \mu \mathrm{g} \mathrm{ml}^{-1}$; kanamycin, $25 \mu \mathrm{g} \mathrm{ml}^{-1}$; streptomycin, $100 \mu \mathrm{g} \mathrm{ml}^{-1}$; tetracycline, $20 \mu \mathrm{g} \mathrm{ml}^{-1}$; chloramphenicol, $30 \mu \mathrm{g} \mathrm{ml}^{-1}$.

Genetic and molecular techniques. Transductions with P1vir, transformations using $\mathrm{CaCl}_{2}$ and conjugations were performed according to standard protocols (Miller, 1992). Gen- 
TTAATTTGATCGCCCGAACAGCAATGTT gadA $202 \quad: \quad: \quad: \quad::: \quad:$ :

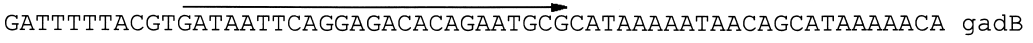

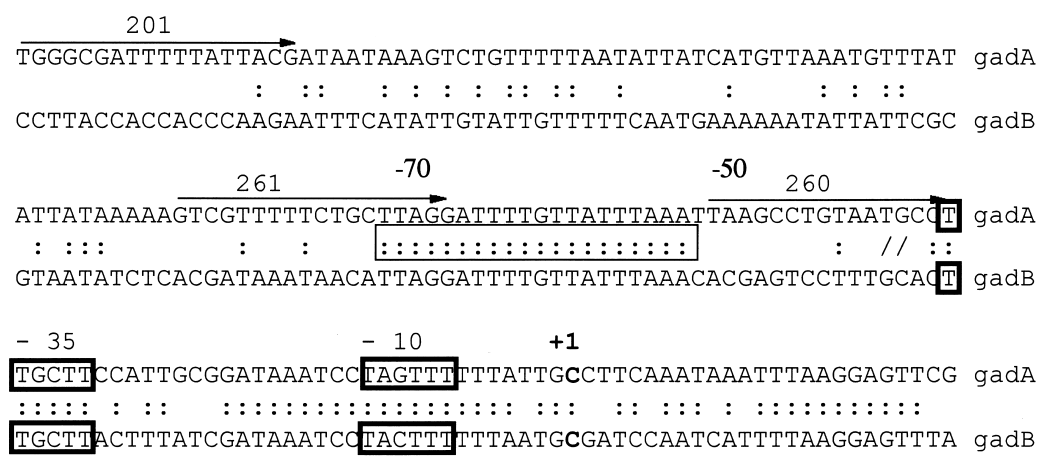

AAATGGACCAGAAGCTGTTAACGGATTTCCGCTCAGAACTACTCGATTCACGTTTTGGC gadA

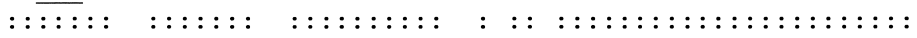

AAATGGATAAGAAGCTAGTAACGGATTTAAGGTCGGAACTACTCGATTCACGTTTTGGT gadB
Fig. 1. Sequence analysis and alignment of $\operatorname{gad} A$ and $\operatorname{gad} B$ promoter regions. The transcriptional start is in bold and indicated by +1 . The ATG start codon is underlined. Primers used to generate gad-lacZ fusions are indicated by arrows. Not shown is oligonucleotide 109, which is located within the coding region of gadA and $B$ at nucleotide +788 (codon 253). Boxes around nucleotides indicate the -10 and -35 recognition sequences. The $20 \mathrm{bp}$ regulatory region is indicated by a box between the $\operatorname{gad} A$ and $\operatorname{gad} B$ sequences. eral DNA manipulations were carried out as described by Sambrook et al. (1989). $\beta$-Galactosidase assays were performed according to Miller (1992). Results presented are representative of triplicate experiments.

Construction of gad-lacZ transcriptional fusions. Transcriptional fusions of gadA and gadB to $l a c Z$ were constructed by cloning PCR-generated fragments of gadA and gadB into the EcoRI site of the pRS551 vector (Simons et al., 1987). The PCR-generated fragments were made using gadA primers 201 (specific to $\operatorname{gadA}$ ) and 109 (5'-CGAACGGTGCCAGGAAGCC-3') and $\operatorname{gadB}$ primers 202 (specific to $\operatorname{gadB}$ ) and 109 (Fig. 1). This created fragments extending from bp -164 to +788 (codon 253) relative to the transcription start for $\operatorname{gadA}$ (pCF384) and from bp -203 to +788 (codon 253) for $\operatorname{gadB}$ (pCF385). These plasmids were then used to introduce the lac Z fusions into a put gene engineered in E. coli as described by Elliott (1992). After XhoI digestion of these plasmids, linearized DNA was transformed into EK298. $\mathrm{Kan}^{\mathrm{R}} \mathrm{Amp}^{\mathrm{S}}$ $\mathrm{Cm}^{\mathrm{s}}$ transformants were obtained following recombination of the plasmid into the chromosome. This resulted in merodiploid strains containing intact gad genes as well as gadAlac $Z$ or gadB-lacZ transcriptional fusions located at the putPA operon (EF615 and EF614 respectively). Similarly, lacZ fusions to truncations of the gadA promoter region were made using oligonucleotides $261 / 109$ (bp -85 to +788 , containing a putative $\mathrm{pH}$ control region) and $260 / 109$ (bp -51 to +788 , containing the -10 and -35 promoter recognition regions but lacking the $\mathrm{pH}$ control region). These fragments were also cloned into pRS551, forming plasmids pCF403 (region from -85 to +788 ) and pCF402 (region from -51 to +788 ). These plasmids were linearized as described above and transformed into EK298 to construct chromosomal fusions.

Purification of nucleic acids, and Northern and Western blotting. Total RNA was isolated from cells grown under different conditions using the RNeasy kit (Qiagen). Aliquots of $5 \mu \mathrm{g}$ were subjected to electrophoresis in a MOPS formaldehyde $1 \%$ agarose gel (Sambrook et al., 1989). The $1.4 \mathrm{~kb}$ gad probe, which hybridizes to both gadA and gadB, was generated by PCR using oligonucleotides 121 (5'-GGAGTTCGAAATGGACCAGAAG-3') and 122 (5'-AGTTTCGGGTGATCGCTGAG-3'), and corresponds to the entire ORF of $\operatorname{gad} A$ or $\operatorname{gadB}$. This fragment was uniformly labelled by including $50 \mu \mathrm{Ci}\left[\gamma^{32} \mathrm{P}\right] \mathrm{dCTP}$ in the PCR reaction. Primer extension analysis of the gad transcriptional start sites was performed as described by Genosys using oligonucleotide 233 (5'-CTCGTCGACACGTGAATCGAGTAGTTC-3'). Western blot analysis was performed using antibodies and conditions described previously (Castanie-Cornet et al., 1999).

\section{RESULTS}

\section{Differential production of Gad in minimal and complex media}

Western blot data presented in Fig. 2(a) (lanes 1 and 2) illustrate that glutamate decarboxylase levels were induced by acid in mid-exponential phase cells grown in minimal media, confirming results from our earlier
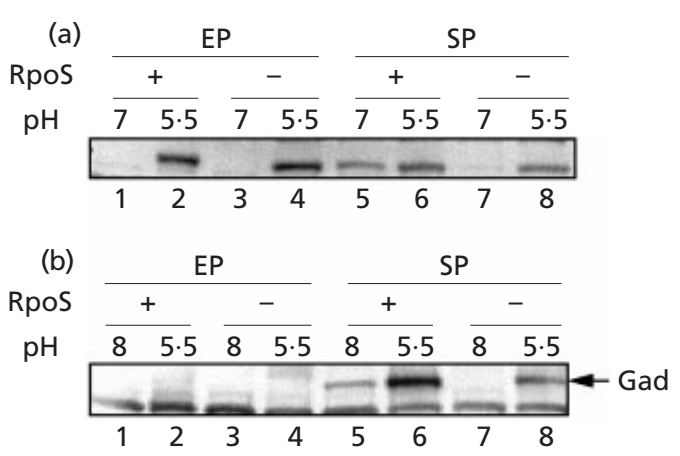

Fig. 2. Western blot analysis of Gad levels. Wild-type (EK227) and rpoS mutant (EF362) strains were grown overnight in LB or EG and diluted $1 / 200$ into fresh medium at specified $\mathrm{pH}$ values. Cultures were grown to $\mathrm{OD}_{600} 0.5-0.7$ (EP, exponential phase) or 1.5-2 (SP, early stationary phase), aliquots were removed and subjected to Western blotting using anti-glutamate decarboxylase antibodies. (a) Gad expression in EG minimal medium at $\mathrm{pH} 7$ and 5.5 (final $\mathrm{pH}$ values). (b) Gad expression in LB complex media buffered at $\mathrm{pH} 8$ and 5.5. The lower band represents a cross-reacting band that is not the product of either gad gene. 


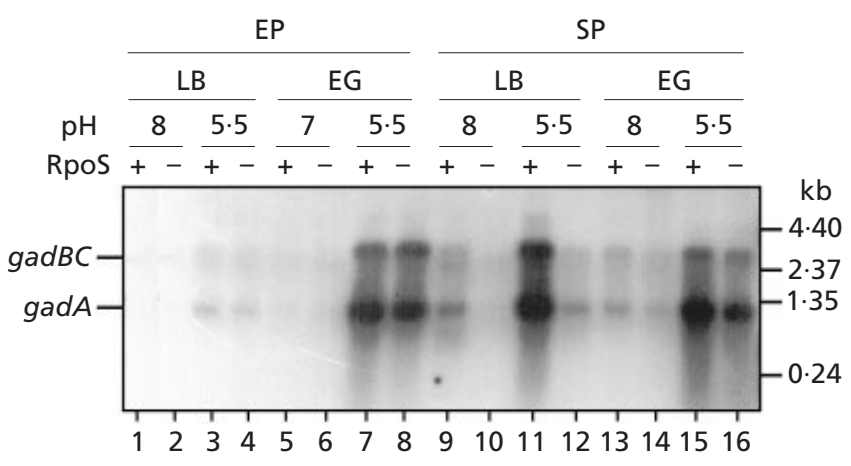

Fig. 3. Northern blot analysis of gad transcription. Wild-type E. coli (EK227) and rpoS mutant (EF362) cultures were grown overnight either in EG minimal medium, $\mathrm{pH} 7$, or in LB. Dilutions (1/500) were made in fresh medium at different $\mathrm{pH}$ values. At $\mathrm{OD}_{600} 0.5$ (EP, exponential phase), $4 \mathrm{ml}$ each culture was harvested, and at $\mathrm{OD}_{600} 1 \cdot 3-1 \cdot 5$ (SP, stationary phase), $2 \mathrm{ml}$ was harvested. Total RNA from these cultures were prepared as described in Methods. Five micrograms total RNA was loaded in each lane, except for lanes 15 and 16 , where only $4 \mu \mathrm{g}$ was loaded. The probe used to detect gad mRNA hybridizes to both the gadA and $\operatorname{gad} B C$ mRNAs.

study (Castanie-Cornet et al., 1999). However, as originally reported by De Biase et al. (1999), we also confirmed that acid induction was not observed in midexponential phase cells grown in complex, rich media (Fig. 2b, lanes 1 and 2). Fig. 2(a) (lanes 3 and 4) demonstrates that the acid induction observed in minimal medium did not require $\sigma^{\mathrm{S}}$ but that stationary-phase induction at neutral $\mathrm{pH}$ in either minimal glucose or complex media was $\sigma^{\mathrm{s}}$ dependent (Fig. 2a, compare lanes 5 and 7 and Fig. 2b, compare lanes 5 and 7). The focus of this study is to determine why acid $\mathrm{pH}$ induces exponential phase cells to produce glutamate decarboxylase in minimal glucose but not in complex LB media and why $\sigma^{\mathrm{S}}$ is only required for stationary phase expression at neutral $\mathrm{pH}$.

\section{Gad production by acid-stressed, exponential phase cells is transcriptionally controlled}

To determine whether the media-dependent regulation exerted over Gad production was due to transcriptional control, we performed Northern-blot experiments on mRNA extracted from wild-type and rpoS mutant strains at different stages of growth and in different media. The results presented in Fig. 3 indicate that both gad $A$ and gadBC behaved in a similar manner, and that transcript levels from each increased more than 10-fold in exponential phase, minimal media grown cells exposed to acidic $\mathrm{pH}$ (Fig. 3, compare lanes 5 and 7). The acid-induced increase in gad mRNA levels was not dependent on $\sigma^{\mathrm{S}}$ in exponential, minimal glucose grown cells (Fig. 3, lanes 6 and 8). In contrast, acid induction of gad expression in complex media occurred mainly in stationary phase cells (Fig. 3, compare lanes 9 and 11) compared to exponential phase cells (Fig. 3, lanes 1 and 3) and was primarily RpoS-dependent (Fig. 3, compare lanes 10 and 12).

\section{Control of gad-lacZ transcriptional fusions}

To gain further insight into gad regulation, gadA-lacZ and gadB-lacZ transcriptional fusions were constructed. These fusions were inserted into the chromosome at the putPA operon, creating merodiploid strains (EF614, gadB-lacZ, and EF615, gadA-lacZ). $\beta$-Galactosidase activities were then measured at different stages of growth in minimal or complex media. Fig. 4(a) presents the results obtained during exponential phase $\left(\mathrm{OD}_{600} 0.5\right)$. In minimal (EG) medium, both fusions showed increased transcription at acidic $\mathrm{pH}$ (sixfold for gadB-lacZ and eightfold for gadA-lacZ). However, during mid-exponential growth in complex (LB) medium, no acid induction was observed. The results were different if activities were measured once cells entered stationary phase $\left(\mathrm{OD}_{600} 1 \cdot 2\right)$. In this case, acid induction in LB medium was evident as 20- and 40-fold increases for gadB-lacZ and gadA-lacZ constructs, respectively (Fig. 4b; EF614 and 615). These results agree with the Western and Northern blot analyses noted above indicating that exponential phase cells exhibit acid induction of the gad genes in minimal but not in complex media.

\section{Acid and stationary phase controls utilize a single transcriptional start site}

De Biase et al. (1999) reported the presence of single transcriptional start sites for $\mathrm{gadA}$ and $\mathrm{gadB}$ in stationary phase-grown cells. We wondered whether the different gad induction profiles observed in minimal and complex media reflected different mRNA transcriptional start sites for stationary phase and acid-induced gad expression. Consequently, primer extension analyses to detect transcriptional start sites were performed on mRNA extracted from exponential phase wild-type cells grown in minimal media at $\mathrm{pH} 5.5$. An antisense oligonucleotide (oligonucleotide 233) able to hybridize with both gadA and gadB was used for this purpose. The start sites, represented as +1 in Fig. 1, were identical to those identified by De Biase et al. (1999), indicating that there is only one start site for each gad gene regardless of the inducing condition (data not shown).

\section{Effect of rpoS on gad transcription}

We have shown previously that rpoS mutations only have a minor effect on glutamate-dependent acid resistance in acid-adapted cells (Castanie-Cornet et al., 1999) and demonstrate here that $\sigma^{\mathrm{S}}$ controls stationary phase but not acid-induced expression of the gad genes. Fig. 4 illustrates that an rpoS mutation (strain EF647) had no effect on the acid $\mathrm{pH}$ induction of gadA transcription in minimal media in either log (Fig. 4a) or stationary phase (Fig. 4b). However, an rpoS mutation did prevent mid-exponential phase transcription of $\operatorname{gadA}$ in neutral $\mathrm{pH}$ minimal media. Thus, midexponential-phase neutral $\mathrm{pH}$ induction of gad in minimal media was most likely due to the early stages of $\sigma^{\mathrm{S}}$ accumulation known to begin in mid-exponential phase and, as will be shown below, to the absence of a 


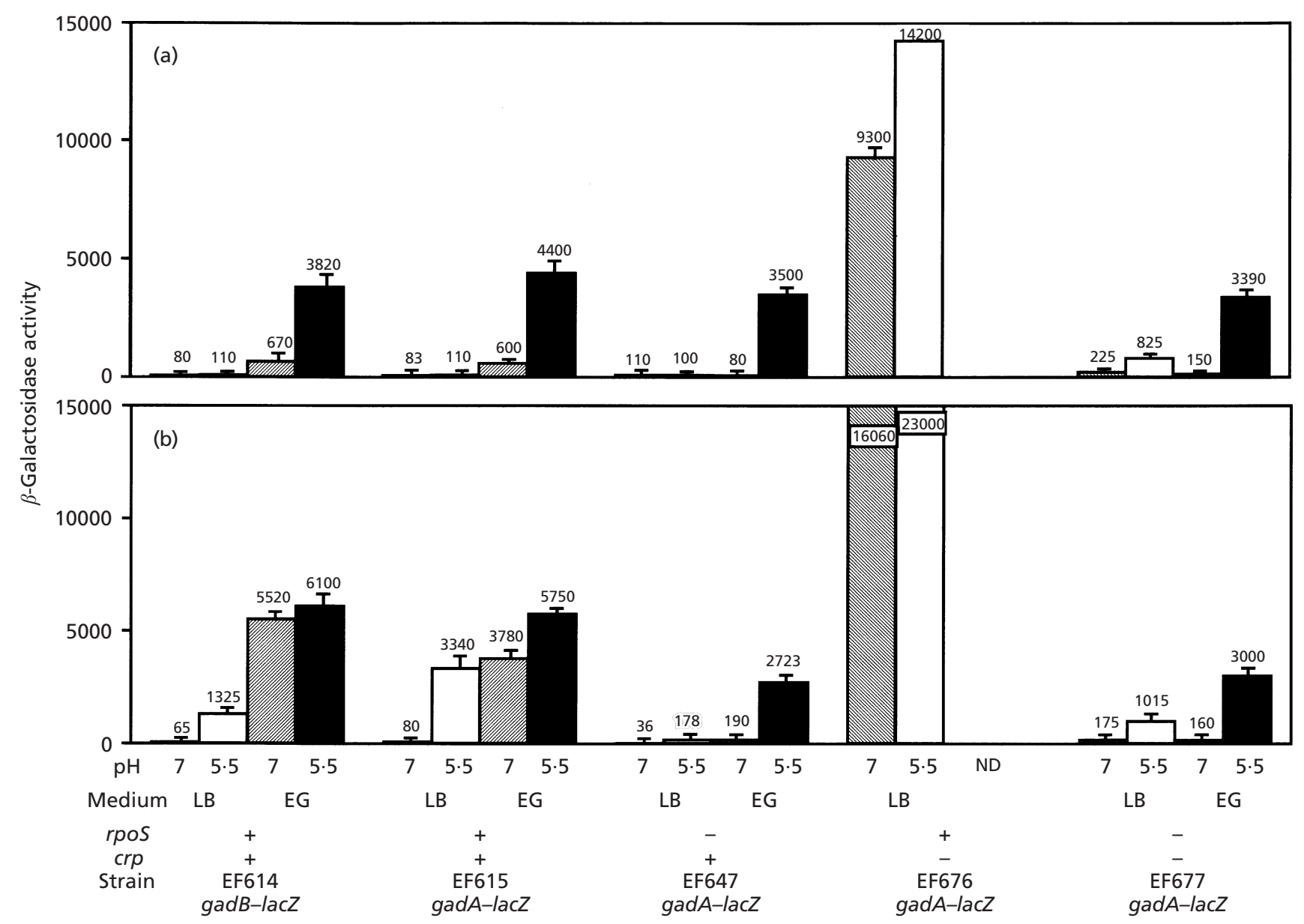

Fig. 4. Effects of media, $\mathrm{pH}, r p o S$ and $c r p$ on gad-lacZ expression. $\beta$-Galactosidase activities were measured during midexponential phase (a) and stationary phase (b) growth. Exponential phase cells were prepared by diluting overnight cultures $(\mathrm{pH} 7) 1: 200$ into fresh medium including LB pH 7, LB pH 5.5 and EG pH 7. For the LB cultures, when the OD ${ }_{600}$ reached $0.5\left(\sim 3 \times 10^{8}\right.$ c.f.u. $\left.\mathrm{ml}^{-1}\right)$, aliquots were assayed for $\beta$-galactosidase activity according to Methods. Early stationary phase cultures were assayed when $\mathrm{OD}_{600}$ reached 1.2-1.5. For expression in minimal media, exponential phase cells were grown to $\mathrm{OD}_{600} 0.2$ at $\mathrm{pH}$, resuspended in $\mathrm{EG} \mathrm{pH} 7$ and $\mathrm{EG} \mathrm{pH} \mathrm{5.5} \mathrm{media,} \mathrm{and} \mathrm{induced} \mathrm{at} \mathrm{that} \mathrm{pH}$ for $2 \mathrm{~h}$ before assay. Final $\mathrm{OD}_{600}$ did not exceed $0 \cdot 4$. ND, Not determined. Experiments were performed in triplicate and individual mean values are presented over each bar. Error bars represent standard error of the mean.

negative regulation present in mid-exponential phase LB cultures that prevents $\sigma^{\mathrm{s}}$-dependent gad expression. It is clear from the results that in minimal media $\sigma^{\mathrm{s}}$ only plays a minor role in directing acid-induced transcription of gad in exponential and stationary phase cells (Fig. 4, EF647, EG). However, in complex media, the rpoS mutation prevented most of the acid $\mathrm{pH}$ induction of gad transcription observed in stationary phase cells (Fig. 4, strain EF647, LB). Consequently, $\sigma^{\mathrm{s}}$ appears to be the major sigma factor used for directing gad transcription for cells grown in complex, but not minimal, media. The dependence on $\sigma^{\mathrm{s}}$ for expression in complex media is consistent with the findings of De Biase et al. (1999). Minimal media cultures, however, must be able to utilize a different sigma factor under acid conditions. Since only one promoter for each gene appears to be involved regardless of the inducing condition, that other sigma factor is most likely to be $\sigma^{70}$, whose recognition sequence is very similar to that of
$\sigma^{\mathrm{S}}$. The fact that mid-exponential phase minimal glucose-grown cells induce gad in the absence of $\sigma^{\mathrm{s}}$ while mid-exponential LB-grown cells do not also suggests that more is involved in regulating gad than simply swapping $\sigma^{70}$ for $\sigma^{\mathrm{s}}$. Either growth in LB subjects the gad genes to a negative regulatory control system not present in minimal glucose cultures or growth in minimal glucose engages an additional positive regulator of gad.

\section{CRP is responsible for the repression in complex media of gadA transcription}

The difference in the induction pattern observed between minimal and complex media indicated that either there is an inhibiting molecule(s) in LB or the glucose present in EG is helping to induce $\operatorname{gad} A$ and $\operatorname{gadB}$ in response to acidic $\mathrm{pH}$. The complex versus minimal media effect on gad expression suggested the involve- 

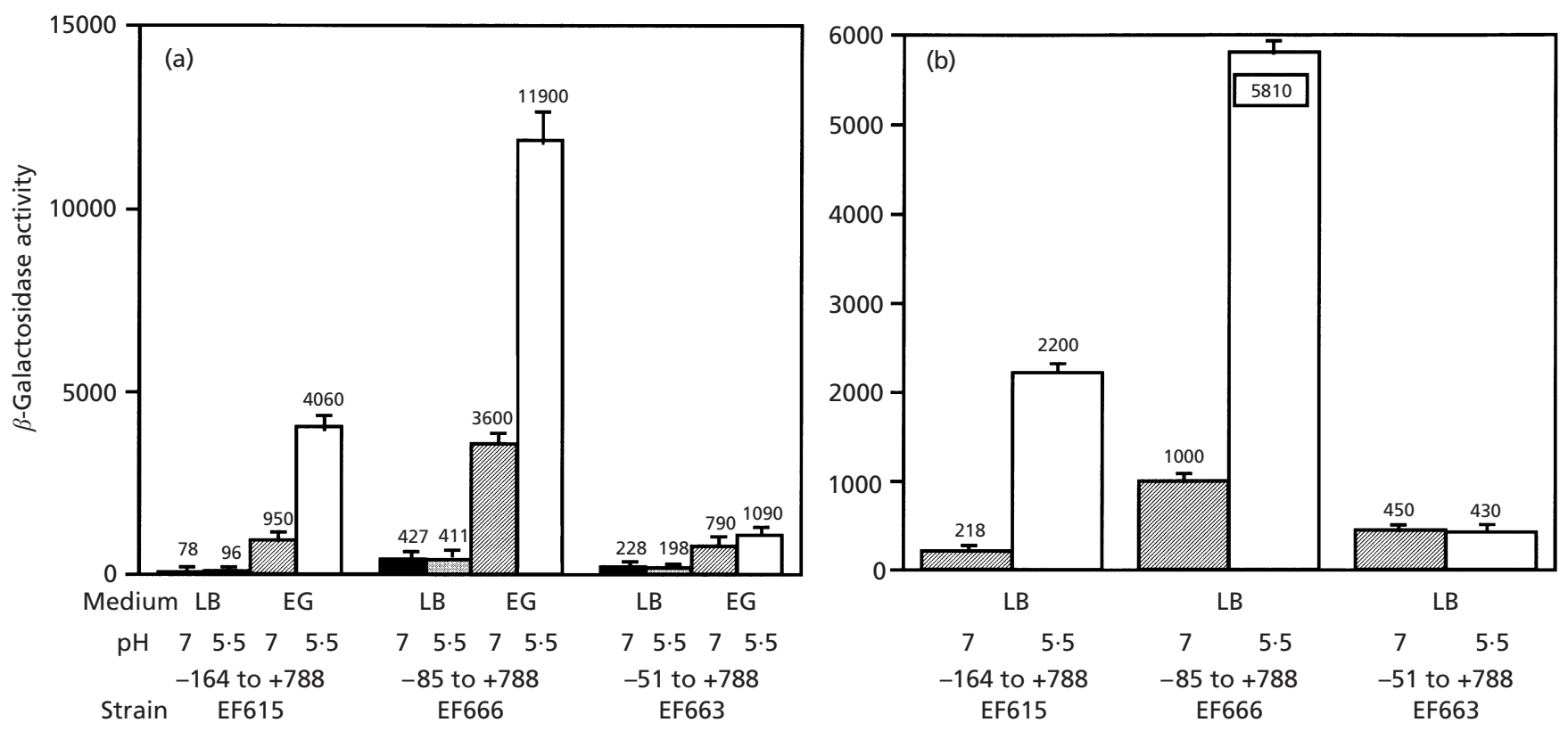

Fig. 5. Identification of the gadA pH response region. Strains EF615 (gad bp -164 to +788 fused to lacZ), EF666 ( -85 to +788 fusion) and EF663 ( -51 to +788 fusion) were grown overnight at neutral pH in LB or EG, and diluted 1/200 in fresh medium, LB pH 7 or pH 5.5 and EG pH 7. For the LB cultures, once the $\mathrm{OD}_{600}$ reached $0.2-0.5$ (exponential phase) (a) and 1.2-1.5 (stationary phase) (b) aliquots were removed and $\beta$-galactosidase activities were assayed. For the minimal EG exponential phase cultures, once the $\mathrm{OD}_{600}$ reached $0 \cdot 2$, cultures were spun and resuspended in an equal volume of EG $\mathrm{pH} 7$ and $\mathrm{EG} \mathrm{pH} 5.5$ and induced for $2 \mathrm{~h}$. The $\mathrm{OD}_{600}$ did not exceed 0.4 . Experiments were performed in triplicate and individual mean values are presented over each bar. Error bars represent standard error of the mean.

ment of CRP and/or cAMP as a negative regulator of gadA transcription in complex LB media. To test this hypothesis, we introduced a $c r p$ mutation into the gadA-lac Z fusion strains and monitored $\beta$-galactosidase activities following growth in minimal and complex media at neutral and acidic $\mathrm{pH}$. The results obtained with strain EF676 (Fig. 4a) confirmed a role for CRP as a negative regulator of $\mathrm{gadA}$ transcription in that the $\mathrm{crp}$ mutation derepressed gadA-lac $Z$ expression in LB grown cells under both $\mathrm{pH}$ conditions and did so independently of growth phase (compare EF676 and EF615 in Fig. 4). These results suggest that in complex media CRP represses gadA transcription and inhibits induction by acidic $\mathrm{pH}$. However, it is unlikely that CRP is directly involved in $\mathrm{pH}$ control since an rpoS crp mutant still exhibits acid induction (EF677, Fig. 4). The high level of expression seen in the crp mutant was essentially due to RpoS-directed transcription since an rpoS mutation reduced gadA-lac expression to near normal levels (strain EF677, Fig. 4).

\section{Identification of a regulatory region in gadA}

The promoter regions of gadA and gadB exhibit considerable homology up to bp -72 relative to the +1 transcriptional start. However, the sequences quickly diverge upstream of position -72 . Of particular interest was a 20 bp block of identity between bp -53 and -72 that we predicted might be involved in the $\mathrm{pH}$ control of gad transcription (Fig. 1). To address this question, transcriptional fusions between $\operatorname{gad} A$ promoter regions containing or lacking this region were made with lacZ. EF663 was constructed to contain a fusion between the $\operatorname{gad} A-51$ to +788 region (missing the $\mathrm{pH}$ regulatory region) whereas the fusion constructed in EF666 contained the region between -85 to +788 , which includes the putative $\mathrm{pH}$ regulatory element. $\beta$-Galactosidase activities presented in Fig. 5 indicate that strain EF663 (lacking the control region) did not exhibit acid or stationary phase regulation. However, EF666 (containing the control region) exhibited both acid and stationary phase controls. This result confirms that acid control centres on the $20 \mathrm{bp}$ region upstream of the -35 site. Curiously, the overall expression of the -85 to +788 fusion (EF666) was two to three times greater than the -166 to +788 fusion (EF615), suggesting that the region between -85 and -166 contains a negative element acting on gadA transcription.

\section{DISCUSSION}

E. coli has maximized the probability of surviving periods of severe acid stress by developing redundant mechanisms for inducing the gad genes. Strategies include $\sigma^{\mathrm{s}}$-dependent stationary phase induction even in the absence of an immediate acid stress and $\sigma^{\mathrm{S}}$ independent acid induction in exponential phase. CRP appears to control whether or not the $\sigma^{\mathrm{S}}$-dependent induction mechanism will operate. Under conditions where cAMP levels are high (e.g. rapid growth in LB), 
CRP appears to prevent $\sigma^{\mathrm{S}}$-dependent transcription of the gad genes. This would be physiologically desirable since under rapid growth conditions it is unlikely that severe levels of acid will be encountered. Upon entering stationary phase or when growing on glucose, cAMP levels are low, thereby allowing $\sigma^{\mathrm{S}}$-dependent gad expression. However, exponential phase minimal glucose grown cells, which possess low levels of cAMP, do not require $\sigma^{\mathrm{S}}$ for acid-induced gad expression. The reason for $\sigma^{\mathrm{S}}$-independent, exponential phase induction of $\mathrm{gad}$ is not intuitively obvious since exponential phase cells that overexpress Gad remain acid sensitive. It is possible that Gad protein produced in exponential phase cells must undergo some form of stationary phase processing in order to become active.

There is a potential, albeit weak, CRP binding site within the $20 \mathrm{bp}$ conserved control region located between $-52 \mathrm{bp}$ and $-73 \mathrm{bp}$ from the transcriptional start site. This site possesses 9 of the 16 consensus nucleotides associated with CRP binding. The presence of this site may explain the ability of CRP to repress $\sigma^{\mathrm{S}}$ dependent expression. The fact that a fusion lacking this region did not exhibit acid induction suggests that an unknown positive regulator also binds to this area. A possible candidate for this regulator is $y$ hiX, which is located downstream of gadA. The YhiX protein appears to be a member of the AraC family of regulators and was indirectly implicated as a gad regulator in gene array studies (Tao et al., 1999). In contrast, $m p p A$, encoding a periplasmic murein peptide-binding protein, was experimentally shown to negatively control Gad synthesis but the level at which this regulation may occur has not been investigated (Li \& Park, 1999).

The main conclusions derived from this study are that 1 ) acid and stationary phase induction of gadA and gadB expression occurs at the transcriptional level;2) a single promoter drives the expression of each gene regardless of the inducing condition; 3) the sigma factor $\sigma^{\mathrm{S}}$ is required for stationary phase induction, but not acid induction, of gad expression; 4) CRP is a negative regulator of $\sigma^{\mathrm{S}}$-dependent gad expression; and 5) a conserved $20 \mathrm{bp}$ sequence located between -52 and $-73 \mathrm{bp}$ in the gadA and gadBC promoters is essential for both acid and stationary phase induction.

\section{ACKNOWLEDGEMENTS}

The authors wish to thank M. Spector, M. Moreno and J. Audia for many helpful discussions. This work was supported by a grant (97-35201-4751) from the US Department of Agriculture.

\section{REFERENCES}

Castanie-Cornet, M.-P., Smith, D., Elliott, J. F. \& Foster, J. W. (1999). Control of acid resistance in Escherichia coli. J Bacteriol $181,3525-3535$.
De Biase, D., Tramonti, A., Bossa, F. \& Visca, P. (1999). The response to stationary-phase stress conditions in Escherichia coli: role and regulation of the glutamic acid decarboxylase system. Mol Microbiol 32, 1198-1211.

Elliott, T. (1992). A method for constructing single-copy lac fusions in Salmonella typhimurium and its application to the hemA-prfA operon. J Bacteriol 174, 245-253.

Gorden, J. \& Small, P. L. C. (1993). Acid resistance in enteric bacteria. Infect Immun 61, 364-367.

Hersh, B. M., Farooq, F. T., Barstad, D. N., Blankenshorn, D. L. \& Slonczewski, J. L. (1996). A glutamate-dependent acid resistance gene in Escherichia coli. J Bacteriol 178, 3978-3981.

Lange, R. \& Hengge-Aronis, R. (1991). Identification of a central regulator of stationary phase gene expression in Escherichia coli. Mol Microbiol 5, 49-59.

Li, H. \& Park, J. T. (1999). The periplasmic murein peptide-binding protein MppA is a negative regulator of multiple antibiotic resistance in Escherichia coli. J Bacteriol 181, 4842-4847.

Lin, J., Lee, I. S., Frey, J., Slonczewski, J. L. \& Foster, J. W. (1995). Comparative analysis of extreme acid survival in Salmonella typhimurium, Shigella flexneri and Escherichia coli. J Bacteriol 177, 4097-4104.

Lin, J., Smith, M. P., Chapin, K. C., Baik, H. S., Bennett, G. N. \& Foster, J. W. (1996). Mechanisms of acid resistance in enterohemorrhagic Escherichia coli. Appl Environ Microbiol 62, 3094-3100.

Loewen, P. C. \& Hengge-Aronis, R. (1994). The role of the sigma factor $\sigma^{\mathrm{s}}(\mathrm{KatF})$ in bacterial global regulation. Annu Rev Microbiol 48, 53-80.

Miller, J. H. (1992). A Short Course in Bacterial Genetics: a Laboratory Manual and Handbook for Escherichia coli and Related Bacteria. Cold Spring Harbor, NY: Cold Spring Harbor Laboratory.

Sambrook, J., Fritsch, E. F. \& Maniatis, T. (1989). Molecular Cloning: a Laboratory Manual, 2nd edn. Cold Spring Harbor, NY : Cold Spring Harbor Laboratory.

Simons, R. W., Houman, F. \& Kleckner, N. (1987). Improved single and multicopy lac-based cloning vectors for protein and operon fusions. Gene 53, 85-96.

Smith, D. K., Kassam, T., Singh, B. \& Elliott, J. F. (1992). Escherichia coli has two homologous glutamate decarboxylase genes that map to distinct loci. J Bacteriol 174, 5820-5826.

Tao, H., Bausch, C., Richmond, C., Blattner, F. R. \& Conway, T. (1999). Functional genomics: expression analysis of Escherichia coli growing on minimal and rich media. J Bacteriol 181, 6425-6440.

Vogel, H. J. \& Bonner, D. M. (1956). Acetylornithase of Escherichia coli: partial purification and some properties. J Biol Chem 218, 97-106.

Waterman, S. R. \& Small, P. L. C. (1996). Characterization of the acid resistance phenotype and rpoS alleles of shiga-like toxinproducing Escherichia coli. Infect Immun 64, 2808-2811.

Received 11 July 2000; revised 22 November 2000; accepted 1 December 2000. 